Ann. Biol. anim. Bioch. Biophys., I968, 8 (2), r95-207.

\title{
INFLUENCE DE LA FRÉQUENCE DES COLLECTES SUR LA DURÉE DU TRANSIT DES SPERMATOZOÏDES DANS LE CANAL ÉPIDIDYMAIRE DU BÉLIER
}

\author{
D. AMIR ( $\left.{ }^{1}\right)$, R. ORTAVANT \\ avec la collaboration technique de A. BURE \\ Laboratoire de Physiologie de la Reproduction, \\ Centre de Recherches vétérinaires et zootechniques, 37 - Nouzilly \\ Institut national de la Recherche agronomique
}

\section{SOMMAIRE}

La durée du transit épididymaire a été mesurée chez des Béliers Ile-de-France au repos sexuel et soumis à différentes fréquences de collecte, après marquage des spermatozoïdes par de la thymidine tritiée.

Les premiers spermatozoïdes marqués font leur apparition dans la tête de l'épididyme le $29^{\mathbf{e}}$ jour et sont trouvés dans le canal déférent le $42^{\mathrm{e}}$ jour après l'injection du précurseur radioactif chez les béliers en repos sexuel.

La durée du transit épididymaire n'est que faiblement influencée par la fréquence de collectes de sperme ; lorsque les béliers sont récoltés 3 fois par jour cette durée diminue dans la plupart des cas de I2-I 3 jours à II jours.

Cinq vagues au moins de spermatozoïdes marqués, d'intensité décroissante, se succédant tous les 2-3 jours ont été détectées dans la partie proximale de la tête de l'épididyme et dans les éjaculats de béliers collectés tous les jours. Chez les béliers collectés une fois par semaine, deux vagues seulement ont été détectées. Ce fait, ainsi que le résultat selon lequel les courbes d'intensité de marquage des éjaculats sous toutes les fréquences de collecte sont du même modèle, suggèrent que l'élimination par d'autres voies des spermatozoïdes non collectés semble aussi efficace que par leur collecte sous une fréquence de 3 fois par jour.

\section{INTRODUCTION}

Lors de leur passage dans le canal épididymaire, les spermatozoïdes strbissent une maturation qui leur permet d'acquérir d'une part la capacité d'être mobiles et d'autre part le pouvoir fécondant (Young, I930; BLANDAU et RUMERY, I96I ; BEDFORD, I966; ORGEBIN-CRIST, I967). Cependant ils ne conservent, dans la queue de l'épididyme et le canal déférent, ces propriétés que pendant un temps limité

(1) Adresse actuelle The Volcani Institute of Agricultural Research, P. O. B. 15, Rehovot (Israël). 
(HAMmOND et ASDEL, I926 ; Young, I930). Aussi un certain nombre de travaux ont-ils été effectués pour déterminer la durée du transit des spermatozoïdes dans 1'épididyme. On a utilisé soit des colorants ou de l'encre de Chine (ToothrLL et Young, I931 ; ClubB, I95I ; Grant, I958 ; GunN, I936) soit des techniques de marquage des spermatozoïles par formation d'anomalies morphologiques (PHILLIPS et MCKENZIE, I934; Glover, I960) ou incorporation de radio-éléments (ORTAVANT, I956 ; DAwson, I95 8 a et $b$; KOEFOED-JOHNSEN, I960 : ORGEBIN-CRIST, I96I-I965 ; Singh, I962; AMANN et al., I965). Ces déterminations ont parfois conduit à des résultats différents. Ainsi chez le Bélier collecté, il a été trouvé que le temps de passage des spermatozoïdes était de 4 à $I_{3}$ ou de $I_{2}$ à $I_{5}$ jours. Ces différences proviennent en partie de l'imprécision des méthodes utilisées.

Devant ces résultats, nous avons repris chez le Bélier l'étude du transit épididymaire des spermatozoïdes et de l'influence de la fréquence des collectes sur celui-ci par le marquage des spermatozoïdes à l'aide de la thymidine tritiée. Ce précurseur permet de différencier et de suivre avec précision les diverses populations de spermatozoïdes marqués (AMANN, KOEFOED-JoHNSEn et LEVI, I965; OrGEBIN-Crist, rg65).

\section{MATÉRIEL ET MÉTHODES}

\section{Animaux}

Vingt-huit béliers Ile-de-France ont été utilisés après vérification de la qualité de leur spermatogenèse à l'aide de collectes de sperme. Leur ration alimentaire était composée de fourrage vert et de $400-500 \mathrm{~g}$ de concentré par animal et par jour.

\section{Technique de marquage}

Vingt-et-un béliers ont reçu unilatéralement et 7 autres bilatéralement dans l'artère testiculaire, une injection de thymidine tritiée, $2 \mu \mathrm{Ci}$ environ par gramme de poids testiculaire estimé, selon la technique de Hochereau, CoUROT et ORTAVANT (1964). Les béliers injectés unilatéralement ont été castrés $28,29,30,32,34,3^{6}, 3^{8}, 4^{\circ}, 4^{2}$ et 77 jours après l'injection. L'épididyme a été subdivisé en 7 parties : tête proximale, médiane et distale, corps et queue proximale et distale. La partie proximale du canal déférent a été également utilisée. Ces différentes sections, après élimination de la majeure partie du tissu conjonctif, ont été broyées avec 2 à $20 \mathrm{ml}$ de sérum physiologique; quelques gouttes des homogénats obtenus ont été étalées sur des lames gélatinées et fixées aux vapeurs de formaldéhyde pendant 20 minutes.

Des échantillons du testicule et de différentes parties de la tête de l'épididyme ont été fixés à l'alcool-formol-acide acétique $(75 / 20 / 5)$, inclus à la paraffine et coupés à $7 \mu$.

Les 7 béliers injectés bilatéralement ont été soumis, 12-I4 jours après l'injection, à des collectes de sperme à l'aide d'un vagin artificiel selon les fréquences suivantes : 3 béliers une fois par semaine, 2 béliers une fois par jour, et 2 autres 3 fois par jour. Les 3 collectes quotidiennes de ces derniers béliers ont été effectuées en 15-30 minutes et les 3 éjaculats mélangés.

Le volume de sperme a été mesuré avec une pipette graduée et la concentration en spermatozoïdes déterminée à l'aide d'un photocolorimètre. Les frottis de spermatozoïdes effectués avec du sperme dilué à une concentration d'environ $100 \times 10^{8} \mathrm{spz} / \mathrm{ml}$ dans du sérum physiologique ont aussi été fixés aux vapeurs de formaldéhyde. Ces béliers ont été castrés 77 jours après l'injection et les différentes parties de l'épididyme traitées comme précédemment.

\section{Technique autoradiographique}

Les coupes histologiques et les frottis ont été revêtus à l'aide d'émulsion Ilford $\mathrm{K} 5$ selon la technique décrite par KOPRIWA et LEBLOND (1962) et exposées pendant 3 semaines à une température $\mathrm{de}+4 \grave{\mathrm{a}}+5^{\circ} \mathrm{C}$. Elles ont été révélées à l'amidol, fixées à l'hyposulfite (FARRAGI, I952) et colorées 
à l'hémalum de Mayer $(15 \mathrm{mn})$. Les coupes provenant des testicules ont été colorées avant le traitement pour l'autoradiographie selon la technique de Feulgen.

Sur chaque frottis 500 à I 300 spermatozoïdes pris au hasard ont été examinés à l'aide d'un objectif à immersion (10o $x$ ) et les grains d'argent au-dessus de chacun d'eux comptés. L'étude du bruit de fond a été effectuée sur des frottis de spermatozoïdes non marqués et a donné les résultats suivants :

\begin{tabular}{l|c|c|c|c|c|c|c|c|c|c|c|c|c|c}
\hline $\begin{array}{c}\text { Nombre de grains } \\
\text { d'argent/spz }\end{array}$ & 0 & 1 & 2 & 3 & 4 & 5 & 6 & 7 & 8 & 9 & 10 & 11 & 12 & Total \\
\hline Nombre de spz.......... & 8284 & 1119 & $\mathbf{1 9 3}$ & 27 & 10 & 5 & & & & & & & & 9639 \\
\hline
\end{tabular}

On s'aperçoit que moins de I p. 100 de spermatozoïdes montrent plus de 2 grains d'argent dans l'émulsion située au-dessus d'eux. Il a donc été décidé de considérer comme marqués, tous les spermatozoïdes ayant plus de 2 grains d'argent. Il a été possible de compter jusqu'à 30 grains d'argent par spermatozoïde sur les frottis préparés à partir de l'épididyme et du canal déférent et jusqu'à 35 grains d'argent sur ceux préparés à partir du sperme éjaculé. Au-delà, il a été impossible de compter les grains d'argent avec une précision suffisante. Les spermatozoïdes marqués ont été dístribués par classes dont les intervalles sont égaux à 3 grains d'argent.

\section{Analyse des résultats}

La comparaison des courbes de fréquence du nombre de grains d'argent au-dessus des spermatozoïdes marqués a été effectuée en utilisant la représentation de LAZAR et GÉRARD- MARCHANT (1965) basée sur le logarithme du nombre maximum de grains d'argent de chaque classe et les probits des fréquences cumulées. La transformation logarithmique permet de rendre normale la distribution de grains d'argent et l'utilisation des probits des fréquences cumulées d'en obtenir une représentation linéaire, après calcul des coefficients de régression.

\section{RÉSULTATS}

\section{Passage des spermatozoïdes marqués à travers l'épididyme} (tableaux I et 2)

Des spermatozoïdes marqués sont observés dans la tête de l'épididyme 29 jours après l'injection du précurseur radioactif. Dès le $30^{\mathrm{e}}$ jour, de tels spermatozoïdes sont rencontrés dans la partie distale de la tête de l'épididyme et parfois dans la partie distale du corps où ils sont constamment trouvés le $32^{\mathrm{e}}$ jour après l'injection. La partie proximale de la queue de l'épididyme en contient les $34^{\mathrm{e}}$ et $36^{\mathrm{e}}$ jours et la partie distale les $3^{\mathrm{e}}$ et $40^{\mathrm{e}}$ jours. Ils apparaissent dans le canal déférent le $42^{\mathrm{e}}$ jour après l'injection. A ce moment-là $\mathrm{I}-2$ p. Ioo des spermatozoïdes comptés dans la tête de l'épididyme sont faiblement marqués (moins de 8 grains d'argent par spermatozoïde). La présence de spermatozoïdes marqués dans la tête de l'épididyme à ces différents moments après l'injection de précurseur radioactif, a été confirmée sur coupes histologiques (fig. I-4).

Ainsi, la durée totale du transit épididymaire est de I2-13 jours pour ces béliers au repos sexuel. Il faut signaler cependant l'existence d'un résultat aberrant; la 


\section{TABLEAU I}

Progression des spermatozoïdes marqués dans l'épididyme de béliers au repos sexuel (p. too de spermatozoïdes marqués)

(Moyenne de 2 béliers)

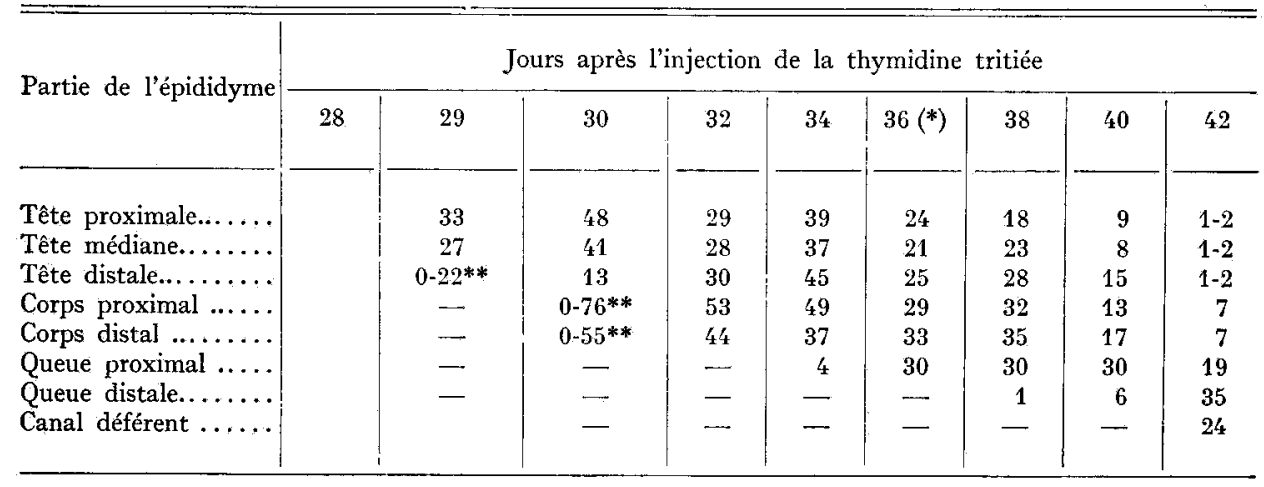

* Un bélier.

** Valeurs chez les deux béliers du groupe.

\section{TABLEAU 2}

Évolution de l'intensité de marquage dans l'épididyme des béliers au repos sexuel (p. Ioo de spermatozoïdes marqués avec plus de $I 7$ grains d'argent) (Moyennes de 2 béliers)

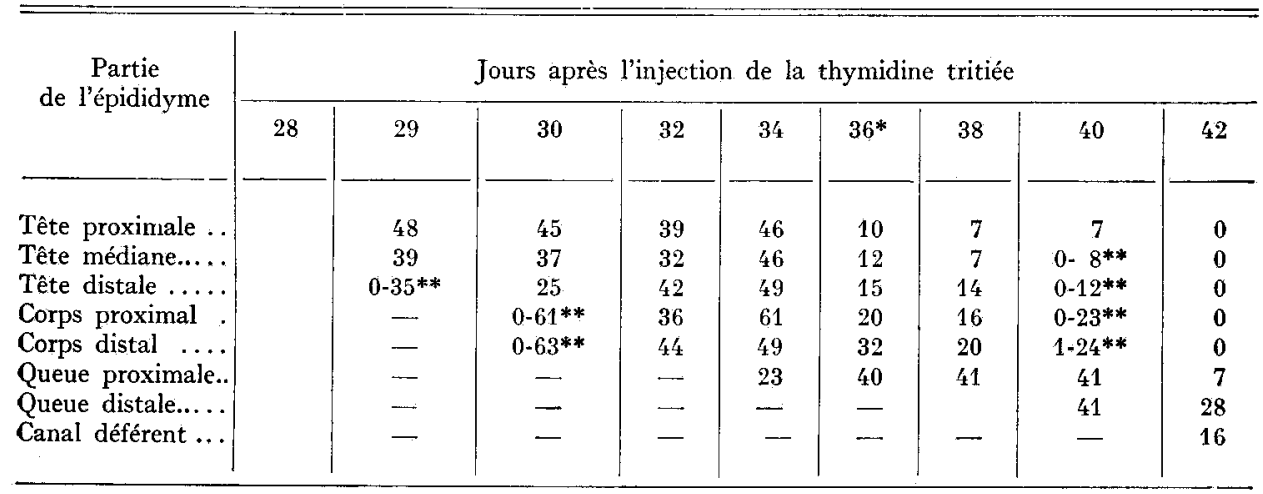

* Au-delà et de deux côtés de cette classe de marquage se trouvent le même nombre de classes plus et moins fortement marqués.

** Valeurs chez les deux béliers du groupe. 

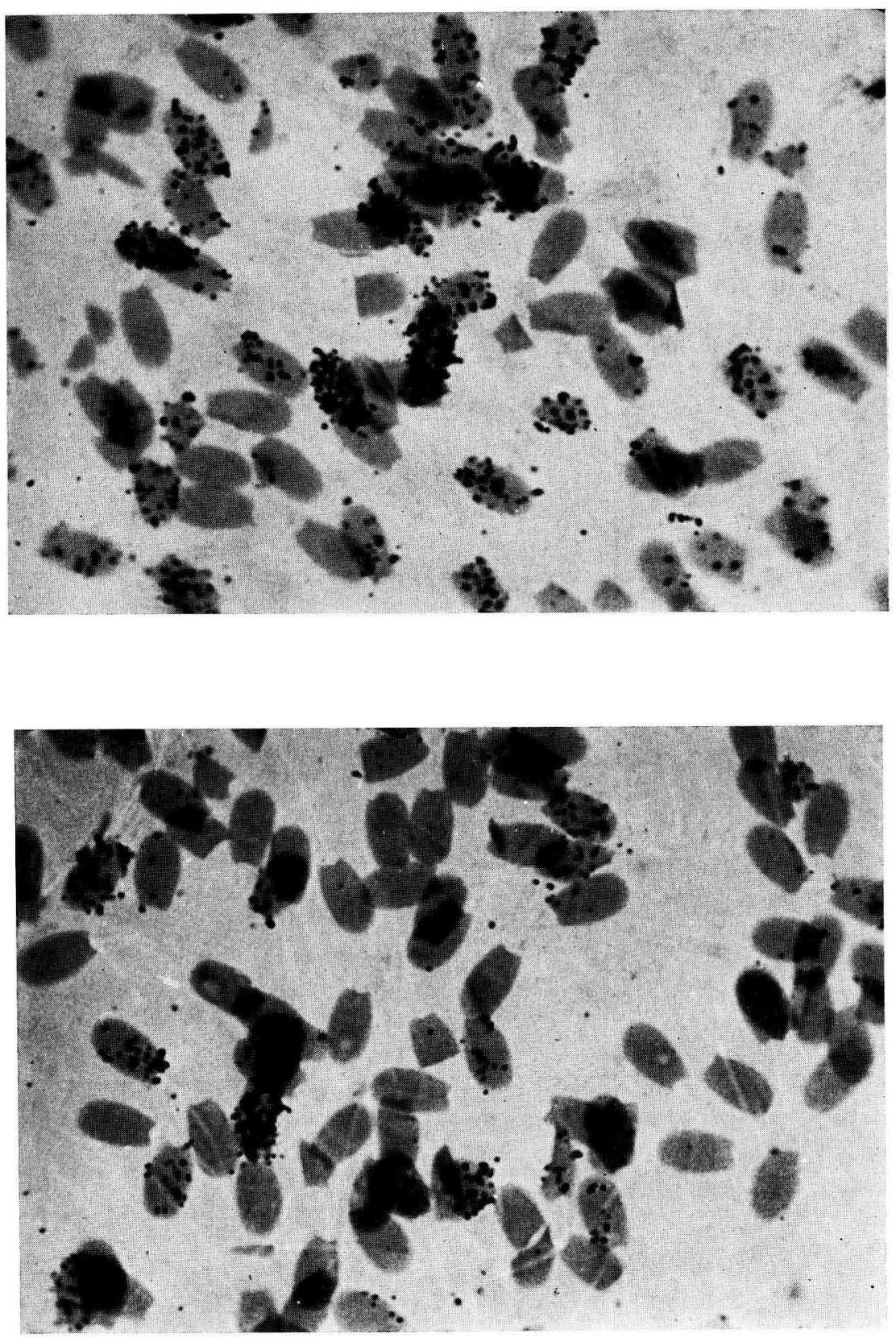

FIG. I. -- Fig. 2. - Coupes histologiques de la partie proximale de la tête de l'épididyme montrant le passage des spermatozoides marqués 29 (fig. I) et 34 jours (fig. 2) après l'injection de Th- ${ }^{-} H$. 

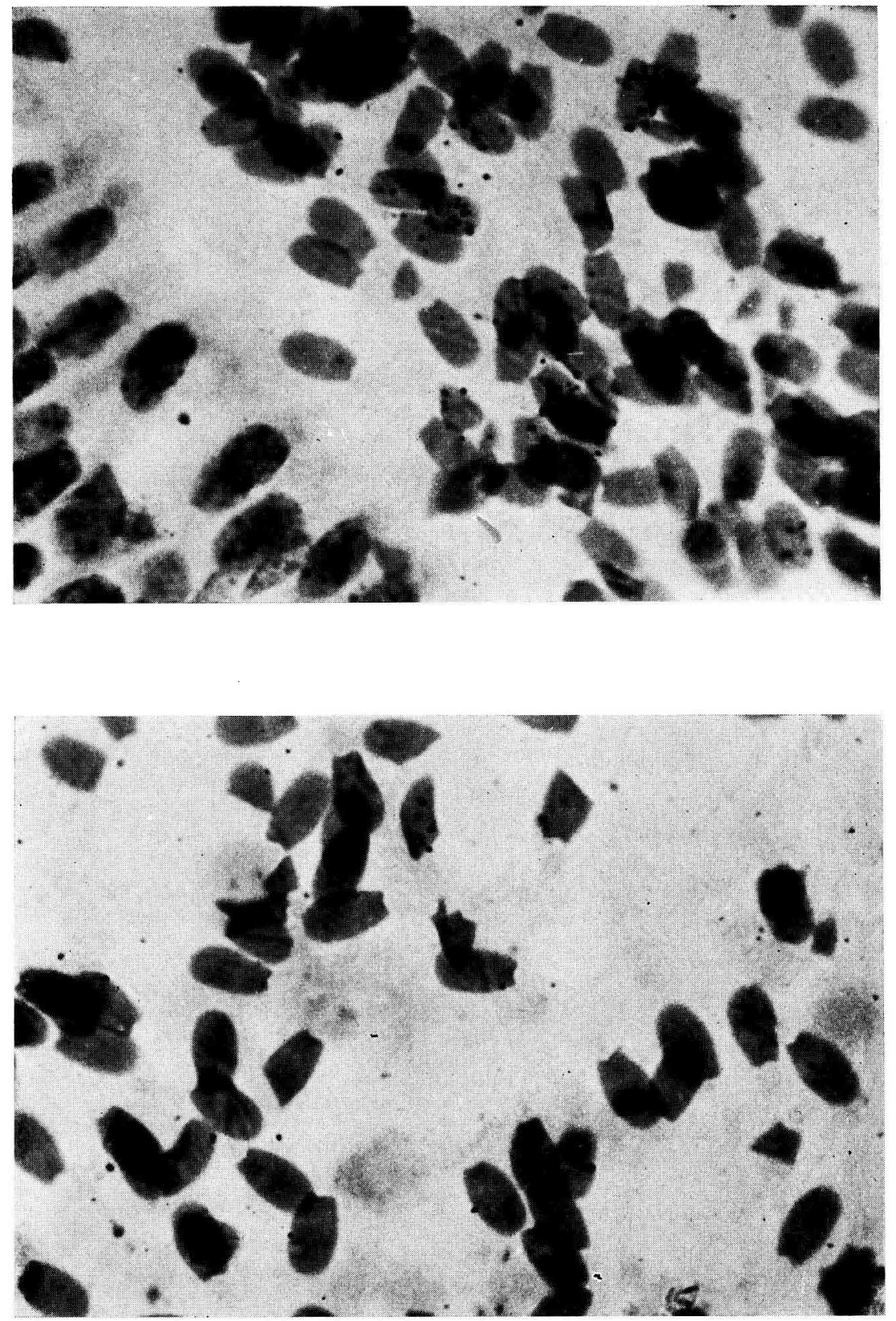

Fig. 3. - FIG. 4. - Coupes histologiques de la partie proximale de la tête de l'épididyme montrant le passage des spermaiozoides marqués 36 (fig. 3 )

et 40 jours (fig. 4) après l'injection de $\mathrm{Th}^{3}{ }^{3} \mathrm{H}$. 

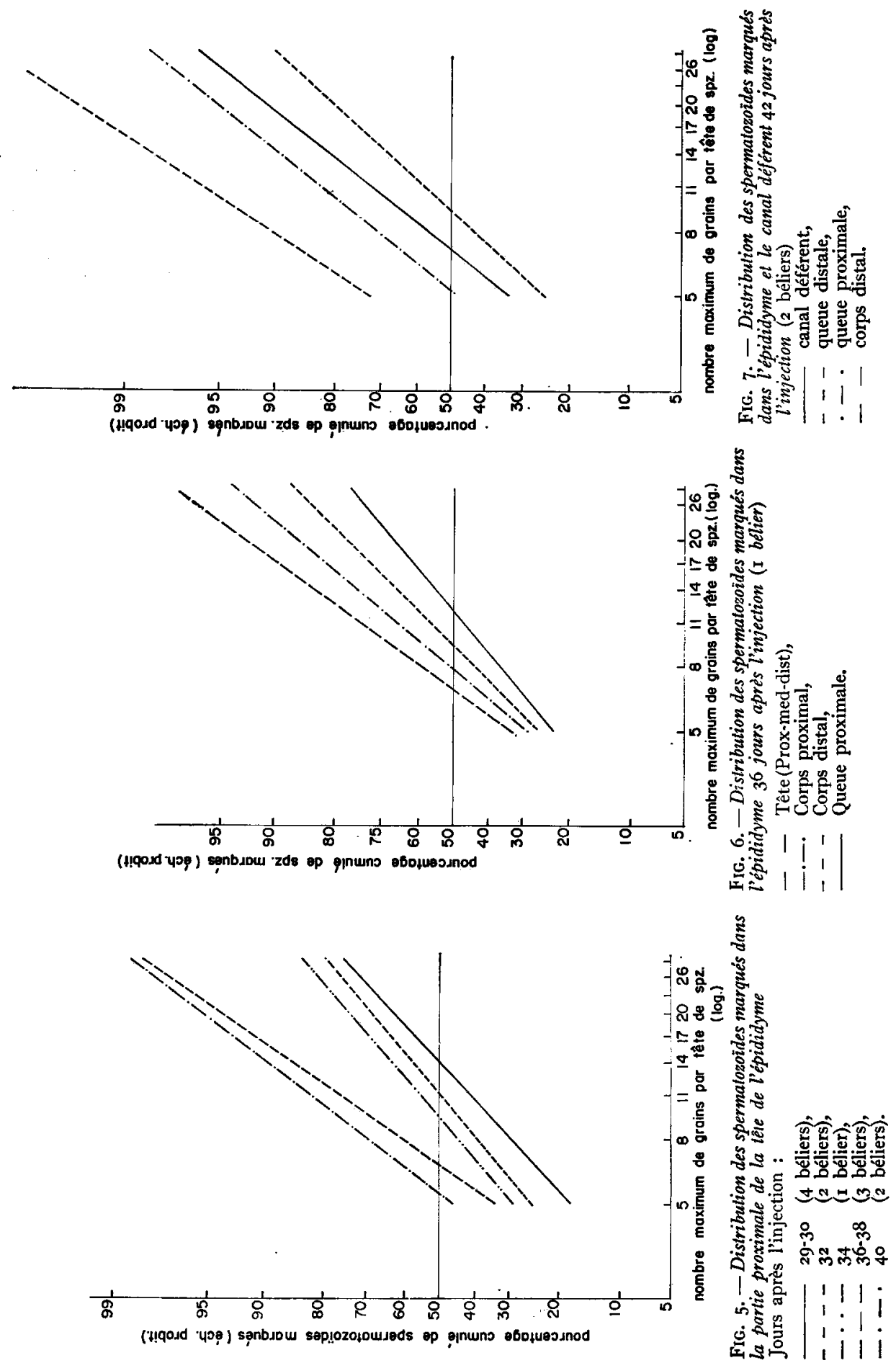
présence de spermatozoïdes marqués dans toutes les parties de l'épididyme et du canal déférent chez un bélier castré, 34 jours après l'injection. La raison de cette anomalie n'a pas encore été trouvée.

Il a été possible de mettre en évidence l'arrivée dans la tête de l'épididyme d'au moins 5 vagues différentes de spermatozoïđes différenciées par une intensité de marquage décroissante (fig. 5). La moitié des spermatozoïdes marqués ont plus de I4, II, 9,7 , et 5 grains d'argent respectivement pour chacune de ces 5 vagues. L'arrivée de ces vagues est espacée de 2 à 3 jours environ, 1a cinquième faisant son apparition dans la tête de l'épididyme le $40^{\mathrm{e}}$ jour après l'injection. On a pu retrouver la progression de ces différentes vagues le long du canal épididymaire : ainsi, 36 jours après l'injection par exemple, on a constaté l'existence de 4 populations différentes de spermatozoïdes marqués respectivement dans la tête, le corps proximal, le corps distal et la queue proximale de l'épididyme (fig. 6).

Il a été observé que les spermatozoïdes trouvés dans le front de la première vague sont moins marqués que leurs suivants immédiats de la même vague. Ainsi le $42^{\mathrm{e}}$ jour les spermatozoïdes du canal déférent sont moins marqués que ceux de la partie distale de la queue de l'épididyme (fig. 7). Ceci peut être dû au fait que les spermatozoïdes arrivant les premiers sont ceux qui se trouvaient en fin de synthèse de l'ADN au moment de l'injection et qui ont moins incorporé de précurseur.

\section{Apparition des spermatozoïdes marqués dans les éjaculats de béliers, collectés à différentes fréquences}

Des spermatozoïdes marqués ont été détectés pour la première fois, le $40^{\mathrm{e}}$ jour après l'injection dans les éjaculats des béliers collectés trois fois par jour, les $4 \mathrm{I}^{\mathrm{e}}$ et $42^{\mathrm{e}}$ jours dans ceux des béliers collectés une fois par jour, le $4 \mathrm{I}^{\mathrm{e}}$ et le $49^{\mathrm{e}}$ jour dans ceux des béliers collectés une fois par semaine.

De même qu'il a été possible de mettre en évidence l'apparition d'au moins 5 vagues successives de spermatozoïdes marqués dans la tête de l'épididyme, de même on constate l'arrivée de 5 vagues successives de spermatozoïdes marqués dans l'éjaculat. Ces 5 vagues se succèdent tous les 2-3 jours comme dans la tête de l'épididyme. Leur intensité de marquage va en décroissant : la moitié des spermatozoïdes marqués de chacune de ces vagues a respectivement plus de 17 , I2, Io, 7-8 et 6 grains d'argent chez les béliers collectés une fois par jour (fig. 8) et II, 9, 8,6 et 4-5 chez les béliers collectés 3 fois par jour. On constate que les spermatozoïdes des 2 béliers collectés 3 fois par jour sont moins marqués que ceux des 2 béliers collectés une fois par jour bien que ces animaux aient reçu approximativement la même dose de $T h-{ }^{3} \mathrm{H}$. La raison de cette différence n'est pas connue.

Par contre, chez les béliers collectés une fois par semaine deux vagues seulement de spermatozoïdes marqués ont été mises en évidence : la moitié des spermatozoïdes marqués ont plus de 9-Io grains dans la première vague et plus de 4-5 grains dans la deuxième. Il est vraisemblable que celles-ci correspondent à la I I $^{\mathrm{re}}$ ou à $1 \mathrm{a} 2^{\mathrm{e}}$ et à la $5^{\mathrm{e}}$ vague détectées dans les éjaculats des béliers fréquemment collectés. En outre l'étude des variations de 1'intensité totale de marquage (nombre total de grains d'argent calculé d'après les autoradiographies pour l'ensemble des spermatozoïdes collectés) montre que celle-ci décroit avec la même vitesse quelle que soit la fréquence de collectes. Il semble donc que, chez les béliers collectés une fois par semai- 
ne, les spermatozoïdes caractérisant les vagues intermédiaires n'apparaissent pas dans les éjaculats. Ces spermatozoïdes sont donc probablement éliminés par d'autres voies.

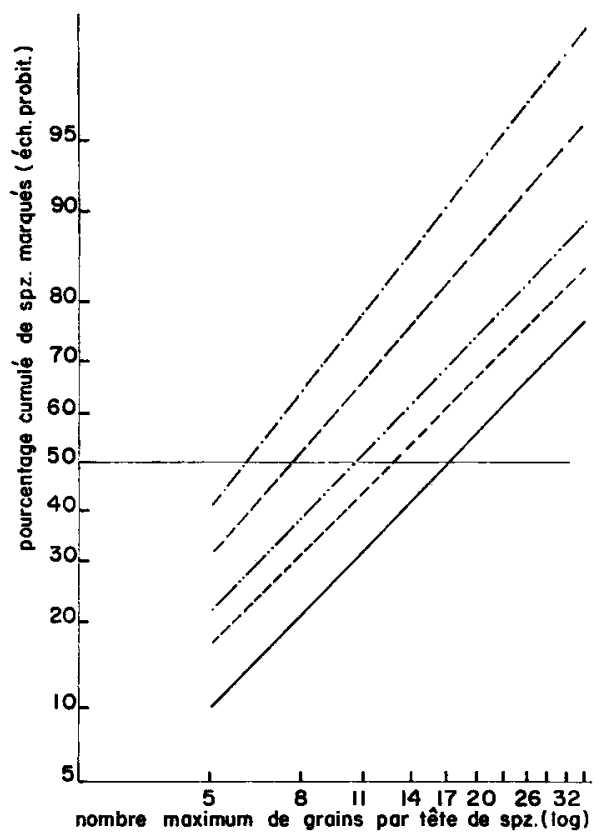

FIG. 8. - Distribution des spermatozoïdes marqués dans les éjaculats des beliers collectés I fois par jour ( 2 béliers)

Jours de l'apparition des spermatozoïdes marqués :

$$
\begin{aligned}
& -\mathrm{I}^{\mathrm{er}-2^{\mathrm{e}}}, \\
& ---3^{\mathrm{e}} \cdot 4^{\mathrm{e}}, \\
& -\cdot-6^{\mathrm{e}} \cdot 7^{\mathrm{e}}, \\
& -\cdots-8^{\mathrm{e}} \cdot 9^{\mathrm{e}}-10^{\mathrm{e}} .
\end{aligned}
$$

La proportion de spermatozoïdes marqués décroît rapidement après le Io jour suivant leur apparition dans l'éjaculat ; il en est de même de leur intensité de marquage. Finalement I p. Ioo seulement des spermatozoïdes examinés étaient marqués par 3 à 5 grains d'argent les $57^{\mathrm{e}}-62^{\mathrm{e}}$ jours chez les béliers collectés 3 fois par jour, le $68^{\mathrm{e}}$ chez ceux collectés une fois par jour et les $6 \mathrm{I}^{\mathrm{e}}{ }^{-} 7^{\mathrm{e}}$ jours chez les béliers collectés une fois par semaine.

L'observation des frottis de spermatozoïdes obtenus des différentes parties de l'épididyme et du canal déférent 77 jours après l'injection a montré que le nombre de spermatozoïdes marqués était parfois légèrement supérieur à $I$ p. Ioo. Cependant il n'est pas possible d'affirmer que ce marquage est significativement supérieur à celui dû au bruit de fond.

\section{DISCUSSION}

Le marquage des spermatozoïdes à l'aide de thymidine tritiée permet d'obtenir des résultats beaucoup plus précis qu'avecle ${ }^{32} \mathrm{P}$, commel'avaient déjà constaté HưP et al. (I96I) chez le Taureau. Ainsi, alors qu'avec le ${ }^{32} \mathrm{P}$ il reste encore Io p. I00 de 
la radioactivité maximum 40 jours après 1'apparition des premiers spermatozoïdes à ADN marqué dans l'éjaculat (KOEFOED-JOHNSEN, I959; ORGEBIN-CRIST, I96I), cette valeur est atteinte en moins de 3 semaines chez le Taureau (HuPP et al., I96r) et de II-I4 jours chez le Bélier avec la thymidine tritiée. Ce fait provient de ce que la thymidine est métabolisée très rapidement; en moins d'une heure la majeure partie est dégradée en métabolites qui ne sont pas incorporés dans 1'ADN (RUBINI et al., I96o ; MONESI, I962).

La thymidine tritiée s'incorpore simultanément dans les noyaux des spermatozoïdes primaires au stade préleptotène et dans ceux des différentes catégories de spermatogonies au cours de la synthèse de l'ADN (MONESI, I962; HochEREAU, r966). Les spermatozoïdes issus de chacune de ces catégories cellulaires sont donc marqués et doivent normalement apparaître dans la tête de l'épididyme en vagues successives séparées par un intervalle de temps égal à la durée de vie de chacune des générations spermatogoniales. Les radioactivités moyennes des spermatozoïdes de deux vagues successives devraient être dans un rapport de 2 puisque les cellules germinales de la deuxième vague ont subi une division spermatogoniale de plus que celles de la première avant de donner naissance à des spermatozoïdes. On a pu effectivement distinguer l'existence d'au moins 5 vagues espacées de $2-3$ jours dans la tête de l'épididyme entre le $29^{\mathrm{e}}$ et le $40^{\mathrm{e}}$ jour après l'injection. La date d'apparition de la première vague (29e jour) est comparable à celle observée à l'aide du ${ }^{32} \mathrm{P}$ (OrTAvaNT, I958), bien que légèrement plus précoce. Cinq vagues ont été également retrouvées dans les éjaculats des béliers collectés $\mathrm{I}$ ou 3 fois par jour avec approximativement les mêmes intervalles de temps. On peut donc supposer qu'il s'agit des mêmes vagues que celles trouvées dans la tête de l'épididyme. Cependant nous n'avons pas pu mettre en évidence l'existence d'un rapport de marquage égal à 2,0 entre les spermatozoïdes de deux vagues successives. Cela provient de ce que, comme nous l'avons dit précédemment, il est difficile de compter les grains d'argent au-dessus d'un spermatozoïde lorsque le marquage est très intense ; cela peut aussi résulter de l'existence de différences entre les durées, donc les intensités, de synthèse de l'ADN des différentes catégories de spermatogonies comme l'a montré HochEREAU (Ig66) chez le Taureau. Il est donc difficile, dans ces conditions, de déterminer si certains spermatozoïdes issus de ces différentes vagues ont pu se mélanger au cours du transit épididymaire comme dans le cas du Taureau ou du Lapin (ORGEBIN-CrIST, I96I et I965). Cependant la durée d'élimination dans les éjaculats des spermatozoïdes fortement marqués étant supérieure à celle de leur apparition dans la tête de l'épididyme, on peut penser qu'un tel phénomène existe, mais à un faible degré.

Le fait que ces différentes vagues n'ont pu être que partiellement détectées chez les béliers collectés une fois par semaine de même que la constance des courbes de variations de l'intensité totale de marquage des spermatozoïdes éjaculés quelle que soit la fréquence des collectes, montrent que chez ces béliers l'élimination, par d'autres voies, des spermatozoïdes non collectés, se produit avec une intensité voisine de celle observée chez les béliers collectés plus fréquemment. Ceci pourrait être en rapport avec les résultats de Lino, BRADEN et TURNBULL (I967) qui ont trouvé dans 1'urine des béliers au repos sexuel un nombre très élevé de spermatozoïdes.

En outre la durée totale du transit des spermatozoïdes dans l'épididyme a été 
influencée par la fréquence des collectes, mais cette influence a été moins importante qu'on ne pouvait le supposer. La durée maximum de ce transit a été de II jours chez les béliers collectés trois fois par jour et de I2-I3 jours chez ceux collectés une fois par jour. Il est plus difficile de calculer avec précision cette durée chez les béliers collectés une fois par semaine compte tenu de l'intervalle entre deux collectes : cependant les premiers spermatozoïdes marqués ont été observés dans l'éjaculat I 2 jours après leur apparition dans la tête de 1'épididyme chez deux béliers et 20 jours chez le troisième. Ainsi l'augmentation des fréquences de collectes n'a pas entraîné, sauf dans un cas, de modifications de durée du transit épididymaire supérieures à I-2 jours. Ces résultats sont à rapprocher de ceux obtenus chez le Taureau par ORGEBIN-CRIST (I96r) et KOEFOED-JOHNSEN (I960)'. Enfin chez les béliers au repos sexuel, une proportion importante de spermatozoïdes marqués a été observée dans la partie distale de la queue de l'épididyme et dans le canal déférent I3 jours après l'apparition de ceux-ci dans la tête de l'épididyme. Ce délai est plus faible que celui obtenu précédemment avec du ${ }^{32} \mathrm{P}$ (ORTAvant, I958). Il est d'ailleurs difficilement explicable puisque les réserves spermatiques épididymaires sont plus élevées chez les animaux au repos sexuel que chez ceux soumis à des collectes (ORTAvanit, I952 et I958; AMANN et AlMQUIST, I962). Nous essaierons d'éclaircir ce point dans un prochain travail.

Reçu pour publication en décembre 1967 .

\section{REMERCIEMENTS}

Ce travail a pu être effectué grâce à une bourse accordée par les gouvernements de France et d'Israël à l'un d'entre nous.

\section{SUMMARY}

THE EFFECT OF EJACULATION FREQUENCY ON THE EPIDIDYMAL TRANSIT OF RAM SPERMATOZOA

In view of the varied results obtained by different workers on the duration of the epididymal transit of ram spermatozoa, depending on the conditions of the work and the methods which were used, we have made a new approach on this problem using tritiated thymidine as a precursor. This DNA precursor enables differentiation the different sperm populations and obtention of more accurate results than does ${ }^{32} \mathrm{P}$.

Twenty one rams Ile-de-France were injected unilaterally into the testicular artery with a dose of about $2 \mu \mathrm{Ci}$ Th- ${ }^{3} \mathrm{H}$ per gram of testicular weight and castrated $28,29,30,32,34,36,3^{8}, 4^{\circ}$ and 42 days after injection. Autoradiographs of sperm smears prepared from the different segments of the ductus epididymidis and the vas deferens show that labelled spermatozoa detected first in the caput epididymidis in the 29th day are present in the vas deferens on the 42nd day after injection. The duration of spermatozoa transit through the ductus epididymidis in rams at sexual rest is therefore 13 days.

7 other rams injected bilaterally as above were submitted to different frequencies of ejaculation : 3 rams once per week, 2 once per day and the 2 other animals 3 times per day. With an ejaculation frequency of once per week and once per day the first labelled spermatozoa were detected in the semen on the 4 rst-42nd day after injection with the exception of one ram ejaculated once per week in which this detection was made on the 49th day. In the two rams ejaculated three times per day the first labelled spermatozoa were detected in the semen on the 4oth day. Thus the duration of the epididymal transit in the rams at sexual rest is accelerated by $1-2$ days only with a frequency of collection of 3 times per day. 
At least 3 waves of labelled spermatozoa were detected in the caput epididymidis and the semen collected once or three times per day during a period of Io-I 2 days. The appearance of the same waves into the two extremities of the ductus epididymidis suggests that if mixing of spermatozoa of different ages takes place in this organ it is not very important at these frequencies of collection. In the rams collected once per week only two were possible to detect the intermediate waves between them being probably eliminated by other means. It seems therefore that the elimination of the non collected spermatozoa in rams ejaculated once per week is as efficient as the collection of three ejaculates per day, this assumption being further confirmed by the identical pattern of the curves of intensity of labelling in the three frequencies of sperm collection.

\section{RÉFÉRENCES BIBLIOGRAPHIQUES}

Amann R. P., ALmQuist J. O., 1962. Reproductive capacity of dairy bulls. VI. Effect of unilateral vasectomy and ejaculation frequency on sperm reserves; aspects of epididymal physiology. J. Reprod. Fertil., 3, 260-268.

AMANN R. P., KoEfoed-Johnsen H. H., Levi E., 1965. Excretion pattern of labelled spermatozoa and the timing of spermatozoa formation and epididymal transit in rabbits injected with thymidine ${ }^{3} \mathrm{H} . J$. Reprod. Fertil., 10, 169-183.

BEDFORD J. M., 1966. Development of the fertilizing ability of spermatozoa in the epididymis of the rabbit. J. Exp. Zool. 163, 319-329.

BlandaU R. J., RUMERY R. E., r96r. Fertilizing capacity of rat spermatozoa recovered from various segments of the epididymis. Anat. Rec., 139, 209 (Abstract).

CluBb R. W., I95I. A study of the epididymal transport of India ink and related epithelial reaction. These, Dept Anat. Univ. Rochester, New York.

Dawson R. M. C., 1958 a. Labelling of bull semen with phosphorus in vivo. Nature, London, 181, ror4IOI 5 .

Dawson R. M. C., I958 b. The labelling of ram semen in vino with radioactive phosphate and (carboxy ${ }^{14} \mathrm{C}$ ) stearic acid. Biochem. $J ., 68,512-519$.

Farragi H., I952. Détection des éléments radioactifs par la technique photographique : Autoradiographie. Comm. En. Alom. France, 27-33.

GLOVER T. D., I 960 . Spermatozoa from the isolated cauda epididymis of rabbits and some effects of artificial cryptorchidism. J. Reprod. Fertil., 1, r21-129.

Grant J. H., I958. The passage of Trypan blue through the epididymis and its uptake by this organ. Proc. Soc. Stud. Fertil. 10, 95-101.

GUNN R. M. C., I936. Fertility in Sheep : artificial production of seminal ejaculation and the characters of the spermatozoa contained therein. Bull. Counc. Sci. Indus. Res. Aust., 94.

Hammond J., AsDelt S. A., I926. The vitality of the spermatozoa in the male and female reproductive tracts. Brit. J. Exp. Biol., 4, I55-185.

Hochereau M.-T., Courot M., Ortavant R., I964. Marquage des cellules germinales du Bélier et du Taureau par injection de thymidine tritiée dans l'artère spermatique. Ann. Biol. anim. Bioch. Biophys., 4, I57-I6I.

HochereaU M.-T., I966. Synthèse de l'ADN, multiplication et renouvellement des spermatogonies chez le Taureau. Colloque Int. Physio. Reprod. Mammifires. Paris (16-19 nov.) Sous presse.

Hupp E. W., Austin J. W., Murphree R. L., I96I. Radioisotope labelling of sperm in bulls and rabbits. J. Anim. Sci., 20, 973 (Abstract).

KOEFOED-JOHNSEN H. H., I959. The influence of srequency of semen collection on the formation time and transport time of spermatozoa in bulls. Roy. Vet. Agric. Coll. Steril. Res. Inst. Ann. Rep., 255-264.

KOEFOED-JOHNSEN H. H., I960. Influence of ejaculation frequency on the time required for sperm formation and epididymal passage in the bull. 'Nature, London, 185, 49-5o.

Kopriwa B. M., LEBLOND C. P., I962. Improvements of the coating technique of radioautography. Histochem. Cytochem., 10, 269-284.

LAZAR P., GERARD-MARCHANT R., 1965. Une technique de représentation graphique des numérations de grains sur autohistoradiographie. Ann. Histochem., 10, 43-52.

Lino B. F., Braden A. W. H., TuRnbull K. E., 1967. Fate of unejaculated spermatozoa. Nature, London, 213, 594-595.

MONESI V., I962. Autoradiographic study of DNA synthesis and the cell cycle in spermatogonia and spermatocytes of mouse testis using tritriated thymidine. J. Cell Biol., 14, I-I8.

ORGEBIN-CRIST M. C., r96I. Recherches expérimentales sur la durée de passage des spermatozoïdes dans l'epididyme du Taureau. Thèse Doct. Sci., Univ. Lyon.

ORGEBIN-CRIST M. C., I965. Passage of spermatozoa labelled with thymidine ${ }^{3} H$ through the ductus epididymidis of the rabbit. J. Reprod. Fertil., 10, 24I-25I.

ORGEBIN-CRIST M. C., 1967. Maturation of spermatozoa in the rabbit epididymis : fertilizing ability and embryonic mortality in does inseminated with epididymal spermatozoa. Ann Biol. Anim. Bioch. Biophys., 7, 373-389.

ORTAVANT R., I952. Recherches quantitatives sur la spermatogenèse et les réserves spermatiques du Bélier. II ${ }^{\mathrm{e}}$ Int. Cong. Physiol. Pathol. Anim. Reprod. Art. Ins. (Copenhagen), 63-69. 
ORtavant R., 1956. Étude sur aa spermatogenèse des animaux domestiques à l'aide du phosphore-32. Conf. Int. Util. pacifique Energie atom. (Geneve), 12, 243-245.

OrTavant R., 1958. Le cycle spermatogénétique chez le Bélier. Thèse Doct. Sci., Paris.

PhILLIPS R. W., McKenzIE F. F., I934. The thermoregulatory function and mechanism of the scrotum. Res. Bull. Mo. Agric. Exp. Sta., 217.

Rubini J. R., Cronkite E. P., Bond V. P., FLIEDNer T. M., i960. The metabolism and fate of tritiated thymidine in man. J. Clin. Invest., 39, 909.

SiNGH G., 1962. Durée de passage dans l'épididyme des spermatozoìdes de Verrat marqué au ${ }^{\text {s2P. }}$ Ann. Biol. anim. Bioch. Biophys., 2, 43.46.

TOOTHILL M. C., YOUNG W. C., 1931. The time consumed by spermatozoa in passing through the epididymis of the guinea-pig determined by India ink injections. Anat. Res., 50, 95-107.

YouNG W. C., I930. A study of the function of the epididymis. III. Functional changes undergone by spermatozoa during their passage through the epididymis and vas deferens in the guinea-pig. $J$. exp. Biol., 8, 151-163. 